Relations industrielles

Industrial Relations

\title{
Labour Relations Institute, Structure and Functions, Geneva, International Labour Office, 1973, 169 pp.
}

\section{Gérard Dion}

Volume 29, numéro 1, 1974

URI : https://id.erudit.org/iderudit/028499ar

DOI : https://doi.org/10.7202/028499ar

Aller au sommaire du numéro

Éditeur(s)

Département des relations industrielles de l'Université Laval

ISSN

0034-379X (imprimé)

1703-8138 (numérique)

Découvrir la revue

Citer ce compte rendu

Dion, G. (1974). Compte rendu de [Labour Relations Institute, Structure and Functions, Geneva, International Labour Office, 1973, 169 pp.] Relations industrielles / Industrial Relations, 29(1), 236-236.

https://doi.org/10.7202/028499ar

Tous droits réservés @ Département des relations industrielles de l'Université Laval, 1974
Ce document est protégé par la loi sur le droit d'auteur. L'utilisation des services d'Érudit (y compris la reproduction) est assujettie à sa politique d'utilisation que vous pouvez consulter en ligne.

https://apropos.erudit.org/fr/usagers/politique-dutilisation/ 
male; les questions financières (capitalisation, dividendes) sont du ressort quasi exclusif de la maison-mère.

La plupart de ces points ont été repris et développés plus tard par la commission Watkins dans son rapport de 1968 qui porte presque le même titre que le livre de Safarian: "Foreign Ownership and the Structure of Canadian Industry $\gg$.

Un mérite, et non le moindre, de l'étude de Safarian est d'avoir été l'inspiration d'un grand nombre d'études plus spécialisées sur différentes questions qu'il avait soulevées dans son livre ; ainsi l'auteur du présent article a mis à profit l'approche et la méthodologie de Safarian pour explorer en détail la question du partage du domaine de la prise de décision entre filiales et maisons-mères.

En conclusion, on ne peut que se féliciter de l'initiative des Presses de l'Université de Toronto; le seule chose que l'on puisse regretter est que Safarian n'ait pas jugé à propos de mettre à jour son travail dont les données remontent déjà à près de 15 ans. L'entreprise plurinationale est en évolution rapide et plusieurs des critiques de Safarian n'ont plus aujourd'hui la portée qu'elles avaient en 1960.

\section{Gérard CORMIER}

Université de Sherbrooke

Labour Relations Institute, Structure and Functions, Geneva, International Labour Office, 1973, 169 pp.

En 1969, la Section de la recherche du Département du développement des institutions sociales du BIT a entrepris une étude sur la structure et les fonctions des instituts de recherche et de formation en matière de relations professionnelles. Achevée en octobre 1971, elle est aujourd'hui publiée.
Après avoir une introduction générale expliquant l'origine, le modèle de l'étude et son étendue, et présentant une typologie des instituts de relations du travail, l'ouvrage aborde en profondeur le genre d'institutions que l'on rencontre en diverses parties du monde. Un chapitre est consacré à chacune des régions suivantes: les Etats-Unis et le Canada; l'Europe; l'Asie, l'Océanie et le Moyen Orient; l'Amérique latine; l'Afrique.

Il existe à travers le monde un nombre considérable d'organismes s'occupant de formation et de recherche en relations du travail sous des noms divers: instituts, centres, départements, etc. Le plus grand nombre est rattaché d'une façon ou d'une autre aux universités. D'autres sont rattachés à des services d'Etat, à des groupements syndicaux ou patronaux. Enfin il y a des organismes entièrement autonomes qui peuvent être subventionnés ou non. Les objectifs aussi varient selon la nature de l'institution. On passe de l'enseignement et de la recherche au niveau universitaire avec l'octroi de diplôme à la formation de personnes déjà engagées dans les relations professionnelles et à l'éducation populaire. Il est assez intéressant de noter que malgré la variété et le caractère des institutions qui sont toujours influencés par la culture, l'histoire et le milieu dans lesquels elles oeuvrent on constate partout certaines constantes quant aux objectifs poursuivis, aux matières enseignées et aux difficultés qu'elles rencontrent.

En publiant cet ouvrage qui permet de faire des comparaisons, le BIT aura rendu un grand service au développement de l'enseignement et de la recherche en relations du travail. Chacun pourra y trouver des éléments pour améliorer les institutions existantes et faciliter la réalisation de leurs objectifs. Le chapitre préparé par Marcel David et consacré à l'Europe est présenté en français.

Gérard DION

Université Laval 